\title{
Fleeing or not: Responsivity of a chased target influences the cognitive representation of the chasing action
}

\author{
Jun Yin ${ }^{1} \cdot$ Man Chen ${ }^{1} \cdot$ Xiaoting Wang ${ }^{1} \cdot$ Xiaowei Ding ${ }^{2}$
}

Published online: 19 March 2018

(C) The Psychonomic Society, Inc. 2018

\begin{abstract}
The chasing action, in which an actor chases a target, is a fundamental activity for the evolutionary shaping of social abilities. Where previous research has emphasized the chaser's role, the current study explored whether the fleeing responsivity of a chased target influences the cognitive representation of the chasing action. We investigated this with a change-detection task, in which a set of chasing actions, either exhibiting or not exhibiting fleeing behavior, were memorized in sequence, and it was tested whether a memorized action reappeared after altering an object's appearance. The results suggest that the target's fleeing responsivity influenced the detection of representation-related changes in the appearance of the involved agents, especially when the appearance of one target was replaced with another (i.e., a new pair, but with the same functional role), showing impaired sensitivity to change for the chasing action (Experiment 1). This effect disappeared, however, when the perceived chasing from presented movements was impaired, while displaying largely the same low-level differences as those present in earlier trials through the use of mirrored chasing (Experiment 2) and setting the faced direction opposite of the moving direction (Experiment 3). These findings suggest that the fleeing responsivity of the chased target can influence the stored representation of the action. This differentiation may be attributed to the stronger construction of social interaction structure for chasing action with fleeing than without, since the fleeing behavior can be deemed a contingency cue for social interaction interpretation.
\end{abstract}

Keywords Chasing $\cdot$ Fleeing $\cdot$ Action representation $\cdot$ Social interaction

The chasing action, in which an actor chases a target, is believed to be a fundamental activity for the evolutionary development of social abilities (Barrett, 2005; Frankenhuis, House, Barrett, \& Johnson, 2013). When observing such an action, even with impoverished display (e.g., if simple geometric shapes are chasing one another), the impressive capability of our visual system extends beyond interpretation as mere physical motions and lets us construe chasing as a high-level social event as the goal of this actor is directed at the target (Blakemore \& Decety, 2001; Woodward, 2009).

Electronic supplementary material The online version of this article (https://doi.org/10.3758/s13414-018-1508-9) contains supplementary material, which is available to authorized users.

Jun Yin

yinjun1@nbu.edu.cn

1 Department of Psychology, Ningbo University, Ningbo 315211, China

2 Department of Psychology and Behavioral Sciences, Zhejiang University, Hangzhou, China
Undoubtedly, the cues generated by the actor itself, such as adjusting its own behaviors, will influence how the visual system constructs and represents this chasing action (Csibra, Bíró, Koós, Gergely, \& Szilvia, 2003; Gao, Newman, \& Scholl, 2009; van Buren \& Scholl, 2017), as this kind of cue is able to signal different action goals (Frankenhuis \& Barrett, 2013; van Buren \& Scholl, 2017). For example, if the direction that an actor faces is inconsistent with its moving direction, the representation of the actor's goal-directness toward the target is impaired (Gao et al., 2009). More than chasing action, studies of eye gaze and body gesture cues show that they influence how the visual system represents the related actions (Adams, 2011; Frischen, Bayliss, \& Tipper, 2007; Gao et al., 2009; Gergely, Nádasdy, Csibra, \& Bíró, 1995; Heider \& Simmel, 1944; Perez-Osorio, Müller, Wiese, \& Wykowska, 2015). Nevertheless, the chasing action must include at least two agents and a display of associated physical activity — being chased — at the minimum. Here, we primarily focus on the simple form of chasing with two agents; hence, the behavioral characteristic of the actor is just one side of the chasing action, and the chased target can respond differently to the actor's chasing. Whether the behavioral characteristics 
of the chased target influence the representation of the chasing action is largely unknown.

Theoretically, to make effective predictions about behavior and provide adaptive benefits for survival, the visual system must understand the specific logic of an action and attune to possible behavioral characteristics indicating fitness-related aspects, such as specific action structures between the actor and the goal (i.e., how the chased target is responsive to the actor's chasing; Frankenhuis \& Barrett, 2013). This adaptive function implies that the representation of observed action would be altered by fitness-related behavioral characteristics, to formulate finer representations of the action and thereby narrow the focus of the prediction space (Barrett, 2008). As to the behavioral characteristics of the target of the chasing action, the most important should be whether the chased target flees or not (i.e., the responsivity of the chased agent; Barrett, 2005). This is because when a target is chased prey, fleeing on time will enhance its survival rate. Previous studies have indicated that vision is sensitive to fleeing-related cues, such as accelerating when the chaser approaches (Frankenhuis et al., 2013; Galazka \& Nyström, 2016; Pantelis et al., 2014). Hence, the fleeing responsivity of the chased target may influence the representation of the chasing action.

The above hypothesis is consistent with one study showing that humans can accurately identify and discriminate the action of pursuit and evasion from the action of leading and following (among other kinds of actions, e.g., guarding and playing; Barrett, Todd, Miller, \& Blythe, 2005). Barrett et al. (2005) recorded the investigated actions in a two-person computer game. A pair of participants were instructed to control a V-shaped arrowhead on the computer screen with a particular intention in mind. In pursuit and evasion action, Participant 1 was asked to pursue Participant 2 while Participant 2 evaded Participant 1 . In contrast, for the leading and following action, Participant 2 was asked to lead Participant 1 and Participant 1 to follow Participant 2. In this manipulation, fleeing responsivity was one critical difference between conditions. The findings showed that participants can differentiate the two actions above chance levels, suggesting that participants differently interpret the action of pursuit and evasion compared with the action of leading and following. Whether the above findings are due to the fleeing cue generated by the chased agent remains unclear, as the controlled chaser in the study of Barrett and colleagues behaved differently between these two conditions. Further, the study used a category judgment task, in which subjects were asked to classify actions into different predefined categories; such tasks can be readily influenced by inferences regarding how "clever" observers think the manipulations should be at diagnosing actions, which may contaminate results from experience-based matching (Gao et al., 2009; Scholl \& Gao, 2013). Particularly when the difference between the actions is subtle, observers may feel obligated to provide an appropriate response. In this way, they might determine or even vary their response on the basis of whatever salient cue in the display matches the content of the experience, even if that cue has only a weak or nonexistent influence on action representation. Given such methodological shortcomings, it is difficult to determine whether human interpretation results in distinct action representations or accurate categorization of the type of chasing action (with fleeing or without).

The current study used an implicit measure to explore whether humans represent chasing actions with fleeing and those without fleeing differently. Participants engaged in a change-detection task, in which a set of chasing actions were memorized in sequence, and it was tested whether a memorized action reappeared by changing the appearance of an object. Our logic is that if humans represent chasing action with and without fleeing behavior differently, it should influence detection of changes related to the content of action representation.

In fact, chasing action, regardless of the responsivity of the chased target, meets the formal and intuitive definitions of social interaction structure (A does X to B; Hinde, 1976). However, the fleeing behavior of the chased target forms its contingent responsivity to the chaser, sometimes referred to as turn-taking, which has been suggested to be an important contingency cue to full-fledged social interaction (A does $\mathrm{X}$ to $\mathrm{B}$ and B responds with Y; Bigelow \& Rochat, 2006; Di Paolo, Rohde, \& Iizuka, 2008; Levinson, 2016; Marsh, Richardson, $\&$ Schmidt, 2009). The function of this cue may emerge from early parent-infant interaction, especially from conversational structure (Bigelow \& Rochat, 2006; Csibra, 2010). Hence, even though a chaser pursuing a target without fleeing behavior can be treated like a social interaction, the strength of its social interaction structure is absolutely weaker than that of the chasing action with fleeing behavior (Bassili, 1976). Hence, in consideration of the change-detection paradigm, the influence of fleeing responsivity on the representation of the chasing action could be determined by examining the sensitivity of change detection, which is implied by the social interaction structure. As previously suggested (Fiske \& Haslam, 1996; Haslam, 1994), people tended to confuse two subordinates occupying the same position in terms of social interaction - that is, these two subordinates are considered equivalent due to their equivalent functional roles in social interaction. Therefore, we predicted that the influence of fleeing responsivity on the representation of chasing action will manifest as follows: if the appearance of a chased agent is changed or rendered onto another chased agent (i.e., a new pair, but with the same functional role), such a change would be more difficult to detect in chasing action with than without fleeing, due to the stronger construction of social interaction structure for chasing action with fleeing.

In the current study, Experiment 1 will test the above prediction by comparing change-detection sensitivity between 
chasing action with and without fleeing. The examination of the explanation of the low-level differences (e.g., statistical correlation between the chaser and target) will first occur in Experiment 2 by manipulating the movement of the chased agent to make the notion of "chasing" subside but keeping almost the same low-level differences between the chasing actions with and without fleeing, as in Experiment 1. Experiment 3 was added to further rule out possible explanations from other low-level factors, such as the distance between two agents, by manipulating the relation between face direction and moving direction. Nevertheless, there is an alternative account that would predict lower sensitivity to detecting new pair changes for the chasing with fleeing than for the chasing without fleeing - that is, the attention/memory mechanism. According to this account, the chasing action without fleeing seems simpler, and therefore may consume less memory resources to memorize accurately. To test this alternative, a new change - swapping the appearance of two agents in the same action event (i.e., swapping appearance; A chasing $\mathrm{B}$ during memorization becomes $\mathrm{B}$ chasing A during test) - was also included. In this case, both agents in the chasing action can be identified by functional roles and relative spatial position markers that do not depend on the target's responsivity. Hence, according to the different action representations account, an effect found in the new pair change would disappear under the swapping appearance change. In contrast, according to the memorization account, the same results should be observed between the new pair and swapping appearance changes.

\section{Experiment 1a}

Experiment 1a manipulated the responsivity of the target in a pursuing action. In a change-detection task, change sensitivity was compared between chasing action with and without fleeing under different changes (e.g., the appearance of the two agents was swapped; the appearance of a chased agent was rendered onto another chased agent), in order to evaluate the influence of the target's responsivity on action representation.

\section{Participants}

On the basis of the results of our pilot studies, we predicted a medium effect size (Cohen's $f^{2}=0.15$; Cohen, 1988) for our experiment design. To ensure adequate power of 0.90 , we performed a power analysis in $\mathrm{G}^{*}$ Power 3 , which determined that at an alpha level of .05, the sample size needed to achieve the predicted effect size was approximately 20 individuals. Twenty adult paid volunteers ( 10 males and 10 females; mean age $=21$ years, age range: $18-25$ years) participated in this experiment; one additional adult was tested but excluded from the sample because their response accuracy did not reach the
$50 \%$ inclusion criterion. All participants had normal or corrected-to-normal vision and no history of neurological disorder. This study was approved by the Research Ethics Board of the Department of Psychology at Ningbo University and granting agencies, and was performed in accordance with the relevant guidelines and regulations. Information sheets were distributed to participants explaining the purpose and procedure of the experiment, after which signed informed consent forms were obtained from all participants.

\section{Apparatus and stimuli}

The stimuli were presented against a black background on a 19 -inch CRT monitor (resolution $=800 \times 600$ pixels; refresh rate $=100 \mathrm{~Hz}$ ) at a $100-\mathrm{cm}$ viewing distance, using a custombuilt script written in The Psychophysics Toolbox for MATLAB (Brainard, 1997).

The visual stimuli were made using the free and open source 3-D creation suite Blender (https://www.blender.org/), and comprised 10-s computer-animated chasing events with fleeing or not, displayed in the center of the screen $\left(11.1^{\circ} \times 8\right.$. $3^{\circ}$; refer to the supplementary videos for details). Each event included a pair of agents: a chaser and a target. These objects were selected randomly and without replacement from ten 3D shapes rendered with distinctive textures to make their appearance as different as possible.

In the chasing action with fleeing, the two agents moved within the border of the ground subtending $11.1^{\circ}$ $\times 11.1^{\circ}$. The initial position of the chaser was $2.8^{\circ}$ below the center of the ground, and the initial position of the target was on the upper right corner of the ground (counterbalanced by display on the upper left corner of the ground), positioned $3.9^{\circ}$ from the center of the ground. Both objects started moving at a speed of $16.7^{\circ}$ per second, and their direction of motion was updated approximately every $50 \mathrm{~ms}$. The target's direction of motion varied randomly on each update, with a uniform distribution within a $90^{\circ}$ angular window centered on its current direction; the chaser's direction was randomly selected on each update, within a $20^{\circ}$ angular window centered on an invisible line connecting the chaser to the target. When the distance between the chaser and the target was less than $2.22^{\circ}$, the target accelerated at $2.08^{\circ}$ per second; otherwise, the speed of both the chaser and target was kept constant at $16.7^{\circ}$ per second.

The animations for the chasing action without fleeing were made with largely the same methods as those for the chasing action with fleeing, except that the speed of both the chaser and target was always kept constant at $19.4^{\circ}$ per second and $13.9^{\circ}$ per second, respectively. All the motions outlined above were presented at a $24.2^{\circ}$ viewing distance and a 3-D perspective view of $57^{\circ}$ (please note that the agents were always oriented toward the moving direction). 


\section{Procedure and design}

Participants sat $100 \mathrm{~cm}$ in front of a CRT monitor with a gray (RGB: 150, 150, 150) background. In each trial (see Fig. 1), a dynamic fixation stimulus was first presented at the center of the screen for $480 \mathrm{~ms}$ and then replaced by a blank background for $300 \mathrm{~ms}$ to $400 \mathrm{~ms}$. Then, within a black wall (subtending $11.1^{\circ} \times 8.3^{\circ}$ ), four memorized items with animations were displayed sequentially, with a $1,000-\mathrm{ms}$ interval between them. The animated test item was subsequently presented, within a red wall (subtending $11.1^{\circ} \times 8.3^{\circ}$ ). Each of the memorized and test animations consisted of a randomly segmented 3-s excerpt from the created 10-s animation. After the test animation disappeared, participants were required to detect, as accurately as possible, whether the test animation differed from the memorized animations. If the test animation was the same as a memorized animation, participants were instructed to press the " $\mathrm{J}$ " button to indicate "no change"; otherwise, the "F" button was pressed to indicate "change." This was followed by an intertrial interval lasting $1,500 \mathrm{~ms}$. If no response was made within $2,000 \mathrm{~ms}$, the program automatically proceeded to the next trial. Response accuracy was recorded, and if the accuracy was less than 50\%, the data from that participant were excluded.

Within a trial, the movements for all 3-s animations were the same (i.e., segmented from the 10-s animation with the same start and end time point), but each memorized animation had a new pair of agents with distinct appearance. In the test animation, the appearance of the two agents was set according to three change types: (1) No change: The same pair of agents as in one of the memorized animations were presented, and the action roles of agents remained identical (i.e., random sampling of a memorized animation displayed as a test animation). (2) Swapping appearance: The same pair of agents used in a memorized animation were presented, but their action roles were swapped (i.e., the object that was the chaser in the memorized animation was the target in the test animation, and vice versa). (3) New pair: The appearance of the chaser in the test animation was the same as that of the chaser in a memorized animation, while the appearance of the target in the test animation was the same as that of a target in a different memorized animation (i.e., the appearance of the agents was sourced from two memorized animations, while the action roles remained unchanged). Due to the use of the same movements across memorized and test animations, participants used the appearance of the two agents to detect change in the animations, irrespective of any knowledge of the change conditions.

To counterbalance the number of trials across change conditions, the number of no-change conditions was double the number of swapping-appearance or new-pair condition. Furthermore, half the trials in the no-change condition were, as a contrast, set as no-swapping-appearance condition, while the other half were considered the no-new-pair condition. In actuality, these two contrast conditions had no critical differences but were coded randomly with equal probability by scripts to meet the requirements of utilization of signaldetection theory (statistical analyses between them did not reveal any significant result for either accuracy or reaction time across the four experiments). All change types were implemented under both chasing with fleeing and chasing without fleeing conditions. Each condition had 20 trials, resulting in a total of 160 trials. The collective trials were equally divided into four blocks with 5-min breaks in between and were presented in pseudorandom order, with the specification that the same condition was never repeated in three consecutive trials.

To examine whether the target's responsivity (with fleeing vs. without fleeing) would modulate the participant's ability to detect changes in agents' appearance, accuracy was determined in terms of signal-detection theory, with "change" as a signal. This allowed us to examine sensitivity $\left(d^{\prime}\right)$ to the change after stripping out the response bias (additional details of the criterion are available in the supplementary information). To avoid infinite hits or false alarms when computing $d^{\prime}$, as suggested by Snodgrass and Corwin (1988), 0.5 was added to each frequency, and the frequency of each condition was divided by $N+1$, where $N$ is the number of trials of that condition. Thus, the experimental design could be simplified as a 2 (target responsivity: with fleeing vs. without fleeing) $\times 2$ (change type: swapping appearance vs. new pair) factorial design. Repeated two-way analyses of variance (ANOVA) were conducted on the resulting $d^{\prime}$ data.

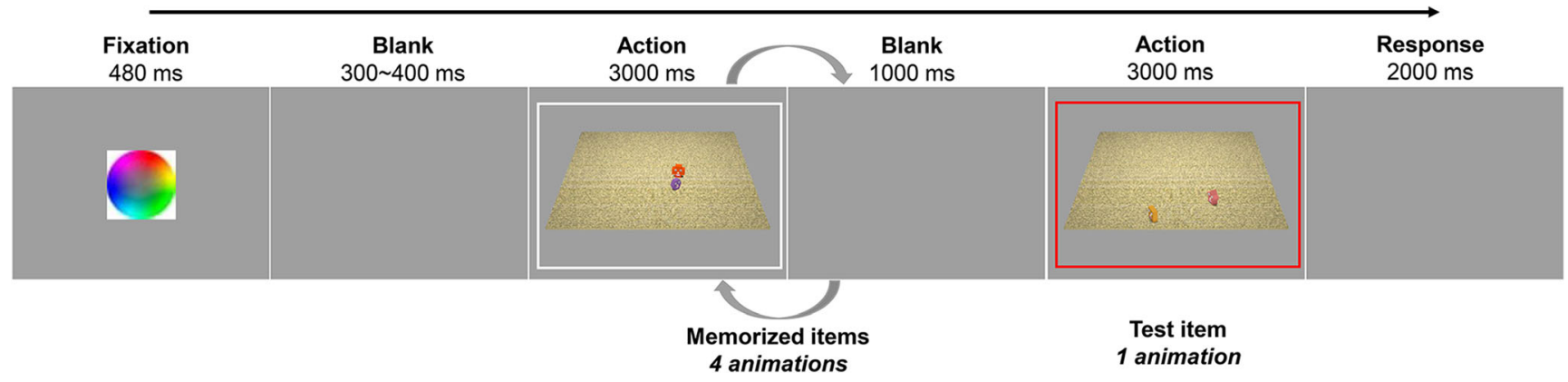

Fig. 1 Illustration of the experimental procedure 


\section{Results and discussion}

The overall $d^{\prime}$ results are presented in Fig. 2a. Analysis of variance revealed that the main effect of target responsivity was significant, $F(1,19)=5.10, p=.036, \eta_{\mathrm{p}}{ }^{2}=.21,95 \% \mathrm{CI}$ $[-0.307,-0.012]$, indicating that participants found it more difficult to detect change when the target behaved with fleeing behavior than without. The main effect of change type was not significant, $F(1,19)=0.74, p=.400, \eta_{\mathrm{p}}{ }^{2}=.04,95 \% \mathrm{CI}$ $[-0.178,0.428]$, while the interaction effect was significant, $F(1,19)=6.38, p=.021, \eta_{\mathrm{p}}^{2}=.25$. The follow-up tests of simple effects showed that when the appearance of the two agents was drawn from different memorized animations (i.e., a new pair), the sensitivity of change detection in the chasing action with fleeing was lower than in the action without fleeing, $F(1,19)=26.11, p<.001, \eta_{\mathrm{p}}^{2}=.58,95 \%$ CI $[-0.503$, $-0.210]$; this difference disappeared when the appearance of the two agents was swapped in a memorized animation (i.e., swapping appearance), $F(1,19)=0.08, p=.776, \eta_{\mathrm{p}}^{2}<.01$, $95 \%$ CI $[-0.237,0.313]$. The results indicate that participants exhibited lower sensitivity to change for the chasing action with fleeing than for the action without fleeing only when a change in appearance occurred between different event structures (i.e., new pairs). This finding is consistent with the hypothesized differences in action representations for chasing events with fleeing and for chasing events without fleeing.
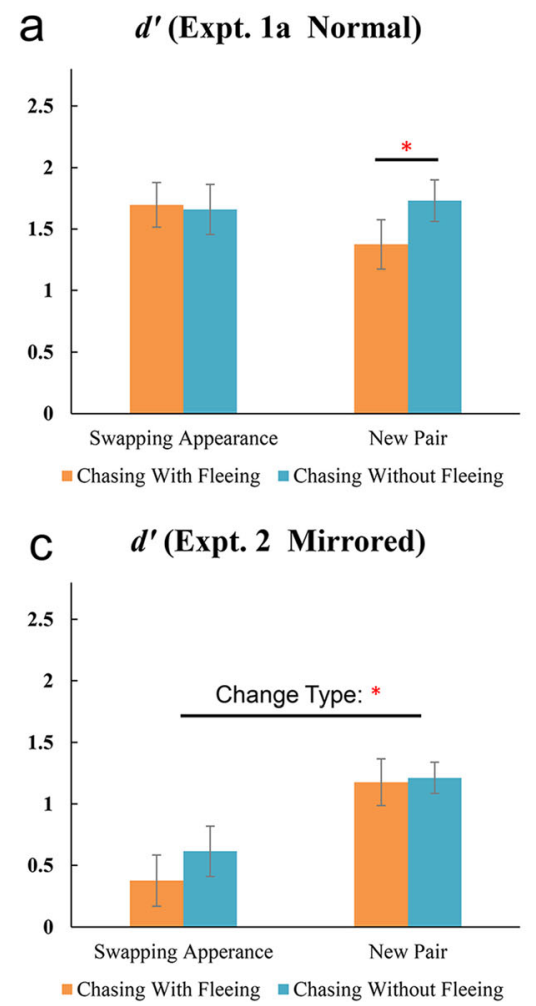

To further support the above conclusion, another 20 participants were recruited and asked to rate subjectively how they perceived the chasing action with or without fleeing. That is, the participants were asked to answer the question "How possible do you think it is that the observed two agents were chasing and being chased?" when watching the chasing action. Chasing likelihood was rated on a scale of 1 (extremely low) to 9 (extremely high). It was found that the perceived likelihood of chasing was higher in the chasing condition with fleeing $(M=6.25)$ than in that without fleeing $(M=4.45), t(19)=2.538, p=.020$, Cohen's $d=0.57,95 \%$ CI $[0.32,3.29]$. Since chasing action without fleeing falls into our daily concept of "follow," another 20 new participants were recruited to answer the question "How possible do you think it is that the observed two agents were following and being followed?" when watching the chasing action. Following likelihood was rated from 1 (extremely low) to 9 (extremely high). In contrast to the evaluation for "chasing," the results for following showed that the perceived likelihood of following was lower in the chasing condition with fleeing $(M=2.60)$ than in that without fleeing $(M=4.45), t(19)=2.547, p=$ .020 , Cohen's $d=0.57,95 \%$ CI $[-3.37,-0.33]$. Therefore, the subjective rating data also support the assertion that humans represent the chasing action with fleeing differently from chasing action without fleeing.
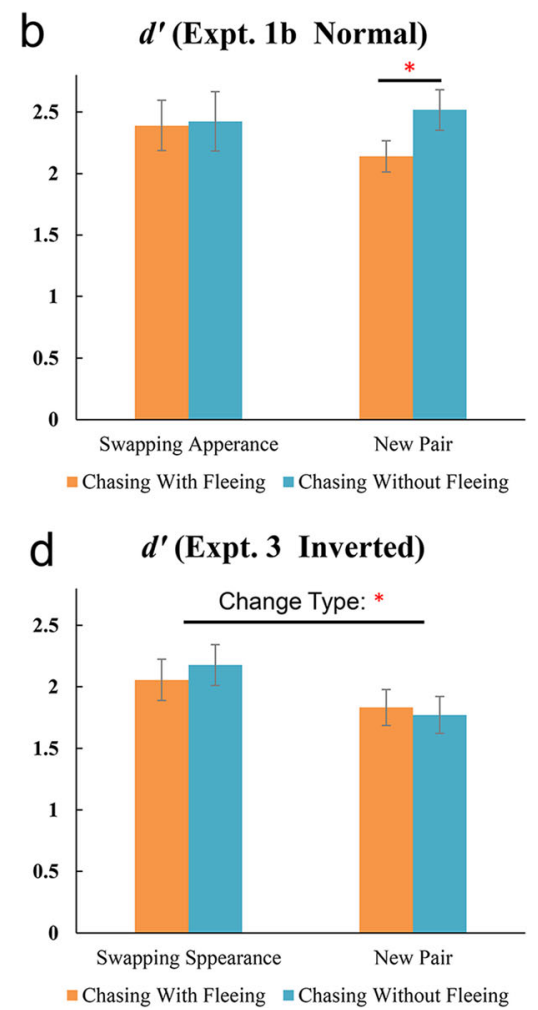

Fig. 2 Sensitivity $\left(d^{\prime}\right)$ of detection of changes in animated displays in Experiment 1a (a), Experiment 1b (b), Experiment 2 (c), and Experiment 3 (d). Error bars indicate standard error 


\section{Experiment 1b}

To verify the findings of Experiment 1a, we conducted Experiment $1 \mathrm{~b}$, which reduced the memory load of memorizing the three animations. The replication of Experiment 1a would support the interpretation that the different sensitivities to detecting a change between types of chasing action (with and without fleeing) when a new pair was presented were not due to high memory load. Thus, all experiment details were identical to Experiment 1a, except that three memory items with animations were displayed, and a new set of student participants was recruited (eight males and 12 females; mean age $=22$ years, age range: 18-25 years). As shown in Fig. 2b, a similar pattern of data as in Experiment 1a was obtained. The ANOVA revealed that participants tended to be less sensitive in detecting change when the chased agent fled than when not, $F(1,19)=4.06, p=.058, \eta_{\mathrm{p}}{ }^{2}=.18,95 \%$ CI $[-0.418,0.008]$. The main effect of change type was not significant, $F(1,19)=$ $0.27, p=.608, \eta_{\mathrm{p}}{ }^{2}=.01,95 \%$ CI $[-0.239,0.397]$, while the interaction effect was significant, $F(1,19)=6.44, p=.020$, $\eta_{\mathrm{p}}{ }^{2}=.25$. The post hoc test of simple effects showed that the sensitivity of change detection in chasing action with fleeing was lower than chasing action without, $F(1,19)=13.10, p=$ $.002, \eta_{\mathrm{p}}{ }^{2}=.41,95 \%$ CI $[-0.593,-0.159]$, when the appearance of the two agents was from different memorized animations (i.e., a new pair), but this effect was not present when the appearance of the two agents was swapped in a memorized animation (i.e., swapping appearance), $F(1,19)=0.06, p=$ $.810, \eta_{\mathrm{p}}{ }^{2}<.01,95 \%$ CI $[-0.322,0.255]$. Hence, the results of Experiment 1 can be replicated with a lower memory load, further suggesting that the responsivity of the chased agent in a chasing action influences action representation.

\section{Experiment 2}

The findings of Experiment 1 were possibly due to different "physical" representations of physical patterns - in particular, the physical properties of each agent and the statistical correlation between the movements of the two involved agents, rather than the "social" difference in action representations between chasing actions with and without fleeing. To further investigate the low-level physical differences, we utilized the experimental design of Gao et al. (2009) and introduced two control conditions, wherein the chaser approaches the mirror image of the target through the center of the display. In this case, the goal of the chase becomes ambiguous in both chasing actions - with and without fleeing - and , accordingly, the impression of a social difference between the agents subsides, while the same low-level differences in action essentially remain. This, in effect, creates mirrored chase events. The "social" explanation would expect that the modulation effect evident in Experiment 1 should disappear when the notion of chasing cannot be extracted.

\section{Method}

Twenty new paid adult volunteers (10 males and 10 females; mean age $=22$ years, age range: $18-25$ years) participated in this experiment; one additional adult was tested but excluded, as their response accuracy was less than $50 \%$. The stimuli and procedure were the same as those outlined in Experiment 1, with the following exception: The trajectories of the target in this experiment were symmetrical in relation to the center of the ground. As such, the chaser now moved toward the point of reflection of the target's location through the center of the ground (please note that in the supplementary videos this symmetry was positioned over the center of the object on the ground in a 3-D scene).

\section{Results and discussion}

Figure 2c presents the overall $d^{\prime}$ results in Experiment 2. The ANOVA revealed that only the main effect of change type was significant, $F(1,19)=17.31, p=.001, \eta_{\mathrm{p}}^{2}=.48,95 \% \mathrm{CI}$ $[-1.051,-0.347]$, suggesting that the sensitivity for detecting change under the new pair condition was higher than that in the swapping appearance condition. Neither the main effect of action type, $F(1,19)=2.28, p=.147, \eta_{\mathrm{p}}{ }^{2}=.11,95 \% \mathrm{CI}$ $[-0.327,0.053]$, nor the interaction effect, $F(1,19)=1.23, p$ $=.282, \eta_{\mathrm{p}}{ }^{2}=.06$, was significant. The results indicate that the influence of action type on the sensitivity of change detection across action structures disappeared with the removal of information-seeking, but the retention of the physical structures from Experiment 1. This finding further suggests that the results of Experiment 1 can actually be attributed to different representations of the chasing action with fleeing or not.

\section{Experiment 3}

Though Experiment 2 ruled out possible physical factors on the individual level as explanations and showed a statistical correlation between agents by presenting a mirrored chase, this operation also changed the distance relation between the two chasing agents. In fact, the distance relation in Experiment 1 could also explain the results of that experiment. Specifically, given that the distance between agents in the chasing action with fleeing was always changing, but that in the chasing action without fleeing was roughly constant, that constant distance might have bound the agent's identity more strongly in a chasing event compared to the varied distance. Accordingly, the new pair change (i.e., appearance change between chasing events) would be more difficult to detect in the chasing action with fleeing than not. Alternatively, perhaps 
the speed difference between two agents might be more noticeable in the fleeing scene (where the speed between chaser and target was set to be different and varied) than in the scene without fleeing (where it was constant), when the visual arrangement of the normal chase was aggregated. In that case, the bond strength between agents' identity and the chasing event (instantiated by the new pair change) would be impaired due to this noticeable difference. The above possibilities can be ruled out by keeping the distance parameters the same as in Experiment 1 but breaking up the chasing relation. As suggested, the agent's directionality is used as a cue to perceive chasing, and misalignment between faced and moving directions impairs perceived chasing (Gao, McCarthy, \& Scholl, 2010; Gao et al., 2009). Hence, the current study set the faced direction opposite to the moving direction to meet control requirements for Experiment 1 (referred to as "inverted chasing"). The influence of fleeing responsivity on action representation would predict that the effect observed in Experiment 1 should not be present under these conditions.

\section{Method}

Twenty new adult paid volunteers (10 males and 10 females; mean age $=21$ years, age range: $17-25$ years) participated in this experiment; two additional adults were tested but excluded, as their response accuracy was less than $50 \%$. The stimuli and procedure were the same as those outlined in Experiment 1, with the following exception: The chaser's face was oriented to the opposite direction of its movement.

\section{Results and discussion}

Figure $2 \mathrm{~d}$ presents the overall $d^{\prime}$ results for Experiment 3 . The ANOVA revealed that only the main effect of change type was significant, $F(1,19)=6.70, p=.018, \eta_{\mathrm{p}}{ }^{2}=.26,95 \% \mathrm{CI}$ $[0.060,0.568]$, showing that the sensitivity of detection of the swapping appearance change was higher than that of the new pair change. Neither the main effect of action type, $F(1$, 19) $=0.19, p=.672, \eta_{\mathrm{p}}{ }^{2}=.01,95 \%$ CI $[-0.182,0.120]$, nor the interaction effect, $F(1,19)=0.482, p=.496, \eta_{\mathrm{p}}^{2}=.03$, were significant. The results show that the influence of fleeing responsivity on the sensitivity of change detection between different chasing events disappeared when impairing perceived chasing and retaining the distance structures of Experiment 1. Hence, the findings in Experiment 1 should be because the representation of chasing action is influenced by fleeing responsivity rather than the low-level confounds. This conclusion was confirmed by the between-experiment analysis, in which, when only considering the new pair change, ANOVA revealed an interaction effect between experiment (Experiment 1 vs. Experiment 2) and action type, $F(1,19)=14.78, p=.001, \eta_{\mathrm{p}}^{2}=.44$.

\section{General discussion}

The results of the current study indicate that the two very similar chasing actions, distinguished by the minor difference of responsivity of the approached target (trying to flee or not), are in fact differentiated in their representations. The fleeing responsivity of the target exerted an influence on detection of representation-related changes in the appearance of the involved agents, especially when the appearance of one target was replaced with that of another, showing impaired sensitivity to change in chasing action with fleeing behavior regardless of memory load (Experiment 1). Furthermore, this effect disappeared when the perceived chasing from presented movements was impaired, while displaying largely the same low-level differences as those present in earlier trials through use of mirrored chase (Experiment 2) and setting the faced direction opposite the moving direction (Experiment 3). As such, the fleeing responsivity of the chased target can influence the stored representation for the action.

Interestingly, the sensitivity of change detection was significantly impaired when the appearance of two agents was swapped in the same action event in Experiment 2. This finding was upheld when compared with the same condition in Experiment 1a, $F(1,19)=18.81, p<.001, \eta_{\mathrm{p}}{ }^{2}=.33$. Such a result for impaired ability may be attributed to the undistinguished markers after removing information-seeking from the task, so that the two agents were almost equivalent in their cognitive construction. In contrast, in Experiment 3, the sensitivity of change detection when the appearance of the two agents was swapped in the same action event was higher than when the appearance of the chaser was replaced by that of another chaser. This enhanced sensitivity tended to be present when comparing the swapping appearance change in Experiment 3 with that in Experiment 1a, $F(1,19)=4.05, p$ $=.059, \eta_{\mathrm{p}}{ }^{2}=.18$. Even the learning of the chasing action was impaired in Experiment 3, where the discriminative markers of relative position and the differentiated functional roles make the swapping appearance change an event sufficiently salient to be detected. Further, directional inconsistency impaired the social interaction structure of the chasing action; this absence of structure diminishes the constraints of a common structure and makes the discriminative marker of distinctive agents more salient, thus showing enhanced sensitivity even relative to Experiment 1a. Furthermore, the impaired (or enhanced) sensitivity of detection of appearance change within an event in Experiment 2 (or Experiment 3) relative to Experiment 1a implies that the meaning of the observed action had been modified, supporting the validity of our manipulation.

The finding of different action representations for chasing action with fleeing versus not raises the further question of what represented content specifically accounts for this 
difference. As discussed in the Introduction, social interaction structure could provide an answer. Categorical information relating to an agent involved in an action structure is said to be defined by its action role (Markman \& Stilwell, 2001; Yin \& Csibra, 2015), which is abstracted from a specific member of a social interaction occupying the same functional position in that interaction (Blok, Newman, \& Rips, 2005; Smith \& Medin, 1981). In our study, fleeing behavior in response to the chaser's approach as a contingency cue induces a stronger social interaction interpretation for chasing action, compared with chasing action without fleeing. That is, due to the different strengths of social interaction structures between these contexts, the abstract action role for agents in the chasing action with fleeing may be more robust and clearer than that without fleeing. Therefore, action type information for the target in the chasing action with fleeing behavior makes it easy to confuse across different events, and thus produces impaired sensitivity to the related change. This conjecture is in accordance with the subjective ratings, in which subjects reported differently how two agents are connected between the chasing action with fleeing and without fleeing. Specifically, chasing action with fleeing was evaluated as having more of a "chase" relation (i.e., one is pursuing and another is evading), while the action without fleeing was rated had more of a "follow" relation. Hence, the influence of fleeing responsivity on the representation of the action may be exhibited as follows: The cue of feeling responsibility has a major impact on the formation of the social interaction structure, which links two agents in a chasing action.

Our findings are consistent with the notion of "islands of competence" proposed by Frankenhuis and Barrett (2013), which suggests that action understanding is centered on domain-specific action schemas and includes different agent concepts relevant to different domains activated by fitnessrelated cues (e.g., fleeing), such as chaser, evader, or follower. The different action representations of chasing action with or without fleeing may thus be the output of domain-specific processes. The domain for one action schema (e.g., "chase") is initiated by the fleeing responsivity cue of the target; otherwise, the observed movement would activate another domainspecific action schema (such as "follow"). Possibly, due to the universal nature of rational reasoning for action (Dennett, 1989; Gergely \& Csibra, 2003), each domain is obeying the rule of rationality to infer a general goal and thereby constraining its specific goal by domain-relevant cues and knowledge.

In any case, fleeing responsivity as a behavioral characteristic of a chased target influences the representation of the chasing action, as demonstrated by the different sensitivities of change detection related to action structure. This differentiation may be due to different social interaction schemas, and could enable humans to produce more precise predictions of an agent's behavior.
Acknowledgements This research was supported by the National Natural Science Foundation of China (31600871) and K. C. Wong Magna Fund in Ningbo University.

\section{Compliance with ethical standards}

Conflict of interest All authors declare that they have no conflicts of interest.

\section{References}

Adams, R. B. (2011). The science of social vision (Vol. 7). New York, NY: Oxford University Press.

Barrett, H. C. (2005). Adaptations to predators and prey. In D. M. Buss (Ed.), The handbook of evolutionary psychology (pp. 200-223). New York, NY: Wiley.

Barrett, H. C. (2008). Evolved cognitive mechanisms and human behavior. In C. Crawford \& D. Krebs (Eds.), Foundations of evolutionary psychology: Ideas, issues, applications and findings (2nd ed.). Mahwah, NJ: Erlbaum.

Barrett, H. C., Todd, P. M., Miller, G. F., \& Blythe, P. W. (2005). Accurate judgments of intention from motion cues alone: A cross-cultural study. Evolution and Human Behavior, 26(4), 313-331.

Bassili, J. N. (1976). Temporal and spatial contingencies in the perception of social events. Journal of Personality and Social Psychology, $33(6), 680-685$.

Bigelow, A. E., \& Rochat, P. (2006). Two-month-old infants' sensitivity to social contingency in mother-infant and stranger-infant interaction. Infancy, 9(3), 313-325.

Blakemore, S. J., \& Decety, J. (2001). From the perception of action to the understanding of intention. Nature Reviews Neuroscience, 2(8), $561-567$

Blok, S., Newman, G., \& Rips, L. J. (2005). Individuals and their concepts. In W.-K. Ahn, R. L. Goldstone, B. C. Love, A. B. Markman, \& P. Wolff (Eds.), Categorization inside and outside the laboratory: Essays in Honor of Douglas L. Medin (pp. 127-149). Washington, DC: American Psychological Association.

Brainard, D. H. (1997). The Psychophysics Toolbox. Spatial Vision, 10(4), 433-436.

Cohen, J. (1988). Statistical power analysis for the behavioral sciences (2nd ed). Hillsdale, NJ: Erlbaum.

Csibra, G. (2010). Recognizing communicative intentions in infancy. Mind \& Language, 25(2), 141-168.

Csibra, G., Bíró, S., Koós, O., Gergely, G., \& Szilvia, B. (2003). Oneyear-old infants use teleological representations of actions productively. Cognitive Science, 27(1), 111-133.

Dennett, D. C. (1989). The intentional stance. Cambridge, MA: MIT Press.

Di Paolo, E. A., Rohde, M., \& Iizuka, H. (2008). Sensitivity to social contingency or stability of interaction? Modelling the dynamics of perceptual crossing. New Ideas in Psychology, 26(2), 278-294.

Fiske, A. P., \& Haslam, N. (1996). Social cognition is thinking about relationships. Current Directions in Psychological Science, 5(5), 143-148.

Frankenhuis, W. E., \& Barrett, H. C. (2013). Design for learning: The case of chasing. In M. D. Rutherford \& V. A. Kuhlmeier (Eds.), Social perception: Detection and interpretation of animacy, agency, and intention (pp. 171-198). Cambridge, MA: MIT Press.

Frankenhuis, W. E., House, B., Barrett, H. C., \& Johnson, S. P. (2013). Infants' perception of chasing. Cognition, 126(2), 224-233.

Frischen, A., Bayliss, A. P., \& Tipper, S. P. (2007). Gaze cueing of attention: Visual attention, social cognition, and individual differences. Psychological Bulletin, 133(4), 694-724. 
Galazka, M., \& Nyström, P. (2016). Infants' preference for individual agents within chasing interactions. Journal of Experimental Child Psychology, 147(Suppl. C), 53-70.

Gao, T., McCarthy, G., \& Scholl, B. J. (2010). The wolfpack effect: Perception of animacy irresistibly influences interactive behavior. Psychological Science, 21(12), 1845-53.

Gao, T., Newman, G. E., \& Scholl, B. J. (2009). The psychophysics of chasing: A case study in the perception of animacy. Cognitive Psychology, 59(2), 154-79.

Gergely, G., \& Csibra, G. (2003). Teleological reasoning in infancy: The naive theory of rational action. Trends in Cognitive Science, 7(7), 287-292.

Gergely, G., Nádasdy, Z., Csibra, G., \& Bíró, S. (1995). Taking the intentional stance at 12 months of age. Cognition, 56(2), 165-193.

Haslam, N. (1994). Categories of social relationship. Cognition, 53(1), 59-90.

Heider, F., \& Simmel, M. (1944). An experimental study of apparent behavior. The American Journal of Psychology, 57, 243-259.

Hinde, R. A. (1976). Interactions, relationships and social structure. Man, 11(1), 1-17.

Levinson, S. C. (2016). Turn-taking in human communication-Origins and implications for language processing. Trends in Cognitive Sciences, 20(1), 6-14.

Markman, A. B., \& Stilwell, C. H. (2001). Role-governed categories. Journal of Experimental \& Theoretical Artificial Intelligence, 13(4), 329-358.
Marsh, K. L., Richardson, M. J., \& Schmidt, R. C. (2009). Social connection through joint action and interpersonal coordination. Topics in Cognitive Science, 1(2), 320-339.

Pantelis, P. C., Baker, C. L., Cholewiak, S. A., Sanik, K., Weinstein, A., Wu, C.-C., ... Feldman, J. (2014). Inferring the intentional states of autonomous virtual agents. Cognition, 130(3), 360-379.

Perez-Osorio, J., Müller, H. J., Wiese, E., \& Wykowska, A. (2015). Gaze following is modulated by expectations regarding others' action goals. PLOS ONE, 10(11), e0143614.

Scholl, B. J., \& Gao, T. (2013). Perceiving animacy and intentionality: Visual processing or higher-level judgment. In M. D. Rutherford \& V. A. Kuhlmeier (Eds.), Social perception: Detection and interpretation of animacy, agency, and intention (pp. 197-229). Cambridge, MA: MIT Press.

Smith, E. E., \& Medin, D. L. (1981). Categories and concepts. Cambridge, MA: Harvard University Press.

Snodgrass, J. G., \& Corwin, J. (1988). Pragmatics of measuring recognition memory: Applications to dementia and amnesia. Journal of Experimental Psychology: General, 117, 34-50.

van Buren, B., \& Scholl, B. J. (2017). Minds in motion in memory: Enhanced spatial memory driven by the perceived animacy of simple shapes. Cognition, 163, 87-92.

Woodward, A. L. (2009). Infants' grasp of others' intentions. Current Directions in Psychological Science, 18(1), 53-57.

Yin, J., \& Csibra, G. (2015). Concept-based word learning in human infants. Psychological Science, 26(8), 1316-1324. 